\title{
Plasmablastic neoplasm in an HIV affected individual and a brief note on its differential diagnosis
}

\author{
Veda Hegde ${ }^{1}$, Kaveri Hallikeri², Venkatesh Anehosur ${ }^{3}$ \\ ${ }^{1}$ Associate Professor, Department of Oral Pathology, SDM College of Dental Sciences and Hospital, Sattur, Dharwad: 580 009, Karnataka, \\ India, ${ }^{2}$ Professor and HOD, Department of Oral Pathology, SDM College of Dental Sciences and Hospital, Sattur, Dharwad: 580 009, \\ Karnataka, India, ${ }^{3}$ Professor and HOD, Department of Oral Surgery, SDM College of Dental Sciences and Hospital, Sattur, Dharwad: 580 009, \\ Karnataka, India
}

\section{A B S T R A C T}

HIV/AIDS predisposes an individual to a variety of malignancies. Lymphomas constitute a group of neoplasm of which are derived from the basic cells of lymphoid tissue, lymphocytes and histiocytes in any of their developmental stages. Thus these neoplasms are closely related and difficult to diagnose based on the histopathology alone. To highlight the importance of immuno-histochemistry in lesions as in the present case and the varied differential diagnosis that is useful to arrive at a diagnosis. We report a case of plasmablastic neoplasm in a young female whose HIV status became apparent only after the histopathologic diagnosis. Oral lesion was the only presenting feature. The lesion was a very aggressive lesion. Diverse differential diagnosis has been illustrated with their specific features which can be valuable to achieve a diagnosis of a particular lymphoma. Although, the histopathology reveals predominantly plasmablasts, it is still not possible to give an accurate diagnosis based on microscopy alone. Thus such lesions have to be differentiated with the help of immuno-histochemistry.

Key words: Acquired immunodeficiency syndrome, Immunodeficiency, Lymphoma, Non-Hodgkin's lymphoma, Diffuse large B cell lymphoma

\section{INTRODUCTION}

With the emergence of AIDS, the development of various neoplasms has been a cause of concern. The association HIV immune- suppression and development of HIV is well established. ${ }^{1}$ The occurrence of neoplasms in HIV infected individuals is associated with immunesuppression rather than the virus itself. However, with the introduction of HAART therapy has significantly altered the outcome of the disease with relatively longer survival time. These categories of cancers constitute three AIDS defining malignancies which include high grade B-cell lymphoma, Kaposi's sarcoma and invasive cervical cancer. ${ }^{2}$ Plasmablastic/plasmacytic differentiation can be found in a wide range of large B cell lymphoma including plasmablastic lymphoma, Immunoblastic diffuse B cell lymphoma, anaplastic lymphoma kinase positive lymphoma and primary effusion lymphoma; plasmablastic plasma cell myeloma, Burkitt's lymphoma with plasmacytic differentiation. ${ }^{3}$

\section{CASE REPORT}

A twenty eight year old female came to our hospital with a huge swelling on the right side of the face (Figure 1) resulting in gross facial asymmetry and extending inferiorly to the floor of the mouth (Figure 2). The swelling was present since the last six months. There was a rapid increase in the size of the lesion since last fifteen days. The swelling was diffuse and measured about $10 \times 15 \mathrm{~cm}$ in its greatest dimension. Intraorally, the painless lesion was reddish white nodular growth obliterating the buccal and lingual vestibule (Figure 3). It extended from 45 to retromolar area and obscuring the crowns of few teeth. 


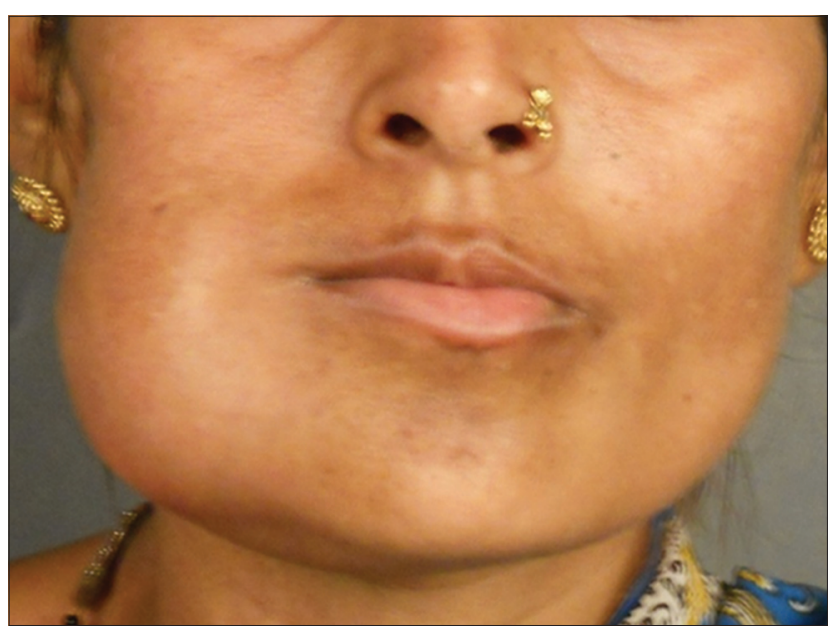

Figure 1: Gross facial asymmetry observed on the right side of the face

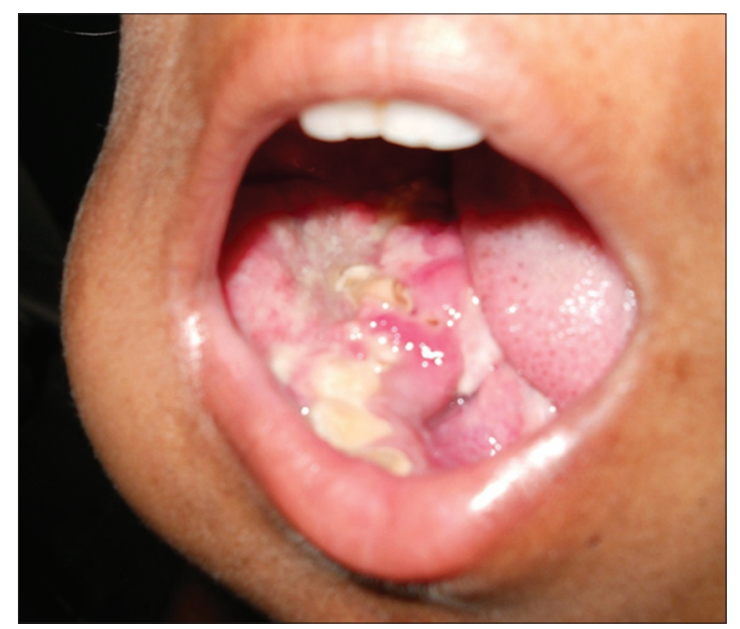

Figure 3: A reddish white nodular growth noted obscuring the crowns of the tooth around the vicinity of the lesion

OPG reveals a diffuse radiolucent lesion associated with the root stumps of 46 and resorption of the alveolar bone. Lymph nodes in the affected area could not be palpated as there was inferior extension of the lesion. Blood and other investigations including bone marrow biopsy could not be done as following incisional biopsy patient was not available and after few months, we lost the patient. Hemopoetic bone marrow defect is evident in the anterior mandibular region (Figure 4). Histopathological examination of the biopsy specimen revealed monomorphic cells consisting vesicular round to ovoid nuclei and eccentrically placed large prominent nucleoli (Figure 5).

\section{DISCUSSION}

Patients with HIV infection have an increased risk of developing malignancies. With the introduction of HAART therapy, there has been a substantial decline in the occurrence of Kaposi sarcoma and non- Hodgkins'

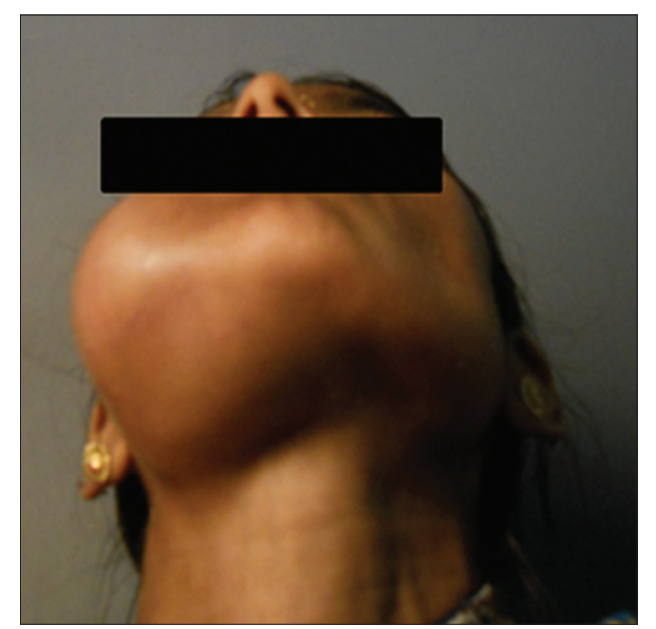

Figure 2: Diffuse swelling extending to the submandibular, sublingual and submental region

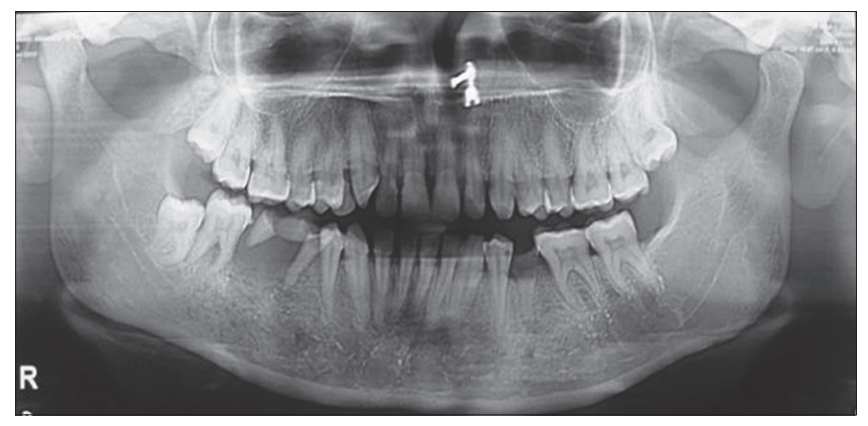

Figure 4: OPG reveals root stumps with evidence of vertical bone loss on the mesial aspect of 47 . Radiolucency is also noted at the anterior apical region of the mandible

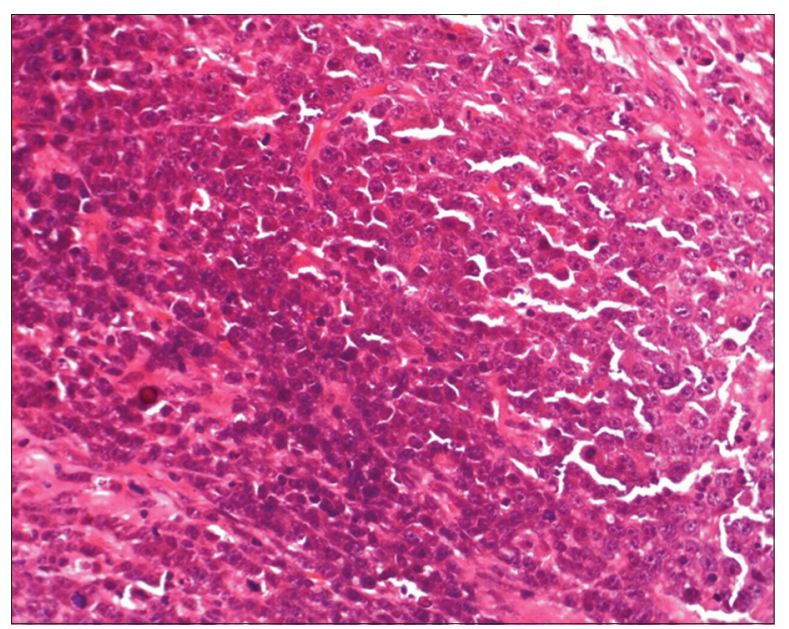

Figure 5: Microphotograph reveals tumour cells in sheets with blast features with minimal connective tissue component. Numerus mitotic figurea and apoptosis also evident. ( H\&E stains, 40x)

lymphoma in patients having HIV infection. ${ }^{4} \mathrm{~B}$ cell lymphomas with plasmablastic features are a heterogeneous group of neoplasm that share many phenotypical features. Many of these have a strong association with HIV infection. 
Identification of these on the basis of histopathology alone is inappropriate. As immuno-histochemistry was not performed on the neoplastic cells in our case, a diagnosis of plasmablastic neoplasm was rendered since it was very difficult to provide a particular diagnosis based on examination of haematoxylin and eosin stained sections alone. However, a suspicion for HIV infection was suspected based on the presence of oral lesion and presence of blast cells on histopathology, which was confirmed to be positive. We also have highlighted the use of specific immunohistochemical markers and other features for the various differential diagnoses that have to be considered in diagnosing lymphomas with plasmablastic/plasmacytic morphology.

\section{Plasmablastic lymphoma}

It is a rare aggressive large B cell lymphoma with immunoblastic/plasmablastic features which often occurs in HIV positive patients. Often involves the oral cavity but can involve nodes, skin, soft tissues and other extranodal sites. It reveals plasmablast morphology consisting of diffuse proliferation of predominantly of large lymphoid cells. These cells are usually positive for Epstien Barr virus and consistently negative for human herpes virus 8 . Most are positive for Epstein Barr Virus latent membrane protein-1 and negative for EBV nuclear antigen-2. The cells are negative for CD45 and CD20 but expressing cytoplasmic IgG and plasma cell related epitopes such as Vs38c and CD138. ${ }^{5}$

\section{Immunoblastic diffuse B cell lymphoma with}

plasmacytoid differentiation

It is difficult to differentiate this neoplasm from plasmablastic lymphoma on the basis of microscopic examination alone although increased apoptosis is evident in the later. The neoplastic cells are negative for CD20 and CD45 and show positive strong reactivity with VS38c antibodies which allows distinction between the two entities. $^{6}$

\section{Primary effusion lymphoma}

This is a rare human herpes virus 8 positive diffuse large B cell lymphoma characterized by lymphomatous effusions into pleural, pericardial or peritoneal cavity occurring in the situation of HIV infection. It is characterised by the presence of large pleomorphic lymphoma cells with cytological features that range between immunoblastic, plasmablastic and anaplastic large cell lymphoma. The cells also have a distinct staining for Leukocyte common antigen CD45 but negative for $\mathrm{B}$ and $\mathrm{T}$ cell markers including CD20, CD19, and CD79a. Neoplastic cells are bcl6 negative and often CD138 positive. Tumour cells are usually co-infected with human herpes virus $8 .^{7}$
Burkitts' Lymphoma with plasmacytico differentiation This also occurs more commonly in immuno-deficient states. The neoplastic cells have a high mitotic rate. When the neoplastic cells may have single central nuclei, its differentiation from plasmablastic lymphoma becomes important. In contrast to plasmablastic lymphoma, these cells have diffuse, strong expression of CD10 and bcl6 and virtually every neoplastic cell is Ki67 positive. Except for endemic Burkitts's lymphoma, a minority of Burkitt's lymphoma are positive for Epstein Barr virus. ${ }^{8}$

\section{Plasmablastic plasma cell myeloma}

It is a highly aggressive neoplasm characterized by postgerminal centre B cell or plasma cell phenotype which closely resembles the malignant cells of plasmablastic lymphoma. Serum monoclonal proteins and/excess light chains in urine, bone involvement with radiographic evidence of lytic lesions, hypercalcemia or anaemia favours the diagnosis of plasma cell myeloma. Epstein Barr virus is not associated with plasma cell myeloma. There is also no strong association of plasma cell myeloma with AIDS. The neoplastic cells exhibit a high proliferative index and are CD138 positive and bcl6 negative.'

\section{ALK positive diffuse large B cell lymphoma}

It has histologic features very similar to plasmablastic lymphoma. Unlike plasmablastic lymphoma, it shows no preponderance to HIV infected individuals. The neoplastic cells typically lack CD20 expression; does not harbour Epstein Barr virus and expresses ALK protein. It typically involves lymph nodes, expresses cytoplasmic $\operatorname{IgA}{ }^{8}$

To conclude, although a variety of $B$ cell lymphomas present with plasmablastic morphology, it is imperative that further analysis are done to allow the neoplasm to be recognized of a particular type. Some of these are seen in a setting of HIV and are very aggressive. Plasmablastic neoplasm is one such group which are characterized by overlapping morphologies and characteristic immunophenotype that can set them apart.

\section{REFERENCES}

1. Radhakrishnan R, Suhas $S$, Kumar RV, Krishnanand G, Srinivasan R and Rao NN. Plasmablastic lymphoma of the oral cavity in a HIV positive child. Oral Surg Oral Med Oral Pathol Oral Radiol Oral Enodol 2005; 100: 725-731.

2. Lee B, Bower M, Newson- Davis T and Nelson M. HIV associated lymphoma. HIV Therapy 2010; 4(6): 649-659.

3. Gorczya W. Atlas of differential diagnosis in neoplastic haematology, $3^{\text {rd }}$ Edition.

4. Flaitz CM, Nichols CM, Walling DM and Hicks MJ. Plasmablastic lymphoma: an HIV associated entity with primary oral manifestation. Oral Oncology 2002; 38: 96-102.

5. Delecluse HJ, Anagnostopoulos I, Dallenbach F, Hummel M, 
Marafioti $T$, Schnerider $U$, et al. Plasmablastic lymphoma of the oral cavity: A new entity associated with HIV infection. Blood1997; 89(4): 1413-1420.

6. Carbone A. Plasmablastic lymphoma of the oral cavity type as a presenting manifestation of HIV infection. Clin Adv in Haemat and Oncol 2010; 8(1): 57-59.
7. Ferry JA. Extranodal lymphoma. pg 62-68. Elsevier Saunders Publication 2011.

8. Vega F, Chang CC, Medeiros L, Udden MM, Cho-Vega JH, Finch CJ, et al. Plasmablastic lymphoma and plasmablastic plasma cell myeloma have nearly identical immunophenotypic profiles. Modern Pathology 2005; 18, 806-815.

Authors Contribution:

All the authors have made substantial contribution to the writing and editing of the article.

Source of Support: Nil, Conflict of Interest: None declared. 